ISSN 1112-9867

Available online at

http://www.jfas.info

\title{
THE RICHNESS AND DIVERSITY OF LEPIDOPTERA SPECIES IN DIFFERENT HABITATS OF THE NATIONAL PARK THENIET EL HAD (ALGERIA)
}

\author{
S. Kacha ${ }^{1}$; M. Adamou-Djerbaoui* ${ }^{1}$; F. Marniche ${ }^{2}$ and W. De Prins ${ }^{3}$ \\ ${ }^{1}$ Departement de biologie, faculté des sciences de la nature et de la vie, Université Ibn Khaldoun \\ ${ }^{2}$ Ecole Nationale Supérieure de vétérinaire - El Alia Alger \\ ${ }^{3}$ Institut Royal des Sciences Naturelles de Belgique
}

Received: 11 February 2017 / Accepted: 18 April 2017 / Published online: 01 May 2017

\begin{abstract}
The diversity of Lepidoptera in several habitats of the National Park Theniet El Had (P.N.T.E.H.) was studied during twelve months of the years 2015 and 2016 in several habitats and totalising 851 specimens belonging to 17 families, 9 super families and 60 species. Among these 31 butterflies and 35 moths were recorded. the clairière (grass fields) turned out to be the most species-rich with 54 species, followed by cédraie with 39 species, the yeusaie with 33 species, the suberaie with 30 species, the zénaie with 29 species and finally the pinaie with only 22 species. The family Nymphalidae was the most dominant one in the parc with $32.48 \%$. The diversity index ( $\mathrm{H}^{\prime}$ and $\left.\mathrm{H}^{\prime} \max \right)$ and the equitability (E) calculated for the 6 types of habitats is $\mathrm{H}^{\prime}=2,74$ bits, $\mathrm{H}^{\prime} \max =4,09$ bits and $\mathrm{E}=0,67$ bits, meaning that the Lepidoptera species are at equilibrium with the different types of habitat which were studied.

Key words: National Park; Theniet El Had; Lepidoptera; Rhopalocera; Heterocera; Diversity (H'); Equitability (E).
\end{abstract}

Author Correspondence, e-mail: kmsoilaz@yahoo.fr

doi: http://dx.doi.org/10.4314/jfas.v9i2.10

\section{INTRODUCTION}

Les papillons et leurs chenilles constituent une source de nourriture indispensable pour un très grand nombre de petits prédateurs comme les oiseaux et les chauves-souris. Sans les papillons 
de nuit, l'ensemble des écosystèmes s'effondrerait rapidement [39]. Les papillons vivent en interaction avec de nombreuses espèces végétales et animales. Ils sont sensibles aux pesticides, aux conditions climatiques, au fonctionnement naturel des milieux [43]. De nombreuses espèces sont facilement identifiables et leurs traits de vie sont bien connus. C'est pourquoi que les lépidoptères sont des bio-indicateurs privilégiés, dont l'évolution des peuplements traduit celle des écosystèmes qu'ils occupent [26]; [47]. Beaucoup de travaux ont été fait au monde sur l'inventaire, la bio-écologie et la répartition des lépidoptères. En Belgique, la diversité des lépidoptères a été étudié par [15], [16], [17]; [18], [19], [20]. [45] s'est intéressé à la taxonomie de nouvelles espèces de lépidoptères en Chine. Les travaux de l'analyse de l'ADN ne sont pas négligeables, compte tenu du fait que la biologie moléculaire est la science la plus étudiée actuellement. Plusieurs auteurs se sont investis dans ce domaine, notamment en Allemagne [32] [33] [34]. [24] en Ukraine et [64] se sont penchés vers les phéromones sexuelles des lépidoptères. En France la biologie des papillons a été synthétisée par [49]. Ces auteurs précisent l'habitat et le comportement des lépidoptères. A propos des travaux effectués sur les Tortricidae il est à mentionner ceux de [38] qui a travaillé en Espagne. A propos des travaux effectués sur les caractéristiques morphologiques externes des papillons, il est à mentionner ceux de [65] [66]. En Afrique peu de travaux sont réalisés dans ce sens ; on cite ceux de [62] qui a traité la biologie des espèces de lépidoptères nuisibles en Tunisie. Au Maroc, [59] a établie un catalogue de lépidoptères. [51] [52] et [58] ont réalisé un travail sur la faune lépidoptérologique de l'Algérie, ayant pour objet la répartition et la description des espèces de lépidoptères. [63] ont décrit la faune lépidoptérologique dans le Hoggar et Tassili. Récemment les travaux de [57] ont fait l'objet d'une synthèse des lépidoptères dans divers agro-écosystèmes de la Mitidja. D'autre part la répartition des insectes dans le parc de Theniet El Had a fait l'objet d'une synthèse d'une étude bulgare [3]. Cette étude précise la présence de peu d'espèces de lépidoptères dans ce parc. Il est à citer aussi les travaux non publiés de [1] [2]; [50]; [9]; [60] et [27]. Les travaux sur les lépidoptères en Algérie sont peu nombreux et pas assez approfondies. Pour cela la présente étude à pour but de réaliser une liste des espèces de lépidoptères dans différents types d'habitats dans une réserve naturelle : Parc National de Theniet El Had.

\section{REGION D'ETUDE}

Le Parc national de Theniet El Had est un massif forestier occupant les deux versants du Djebel El Meddad (Montagne des cèdres). Il est situé à $173 \mathrm{~km}$ au sud-ouest d'Alger et à peu 
près à $48 \mathrm{~km}$ du chef-lieu de la wilaya de Tissemsilt. Le parc s'étend sur une superficie de 3.424 ha. Il est partie prenante de l'Ouarsenis. Ensemble, ils constituent la chaîne sud de l'Atlas tellien. L'Ouarsenis est le principal chainon du Tell occidental. Le parc se situe entre les coordonnées géographiques : $35^{\circ} 49^{\prime} 41^{\prime \prime}$ ' et $35^{\circ} 54^{\prime} 04^{\prime \prime}$ de latitude Nord et $01^{\circ} 52^{\prime} 45^{\prime \prime}$ ' et 02 $02^{\prime} 04^{\prime}$ ' de longitude Est (Fig. 1). La forêt des cèdres de Theniet El Had fut la première espace protégé en Algérie. Elle fut proclamée parc national par l'arrêté gouvernemental du 03/08/1923. Passant sous la règlementation algérienne, elle est décrétée une nouvelle fois parc national sous le $\mathrm{n}^{\circ}$ 459/83 du 23/07/1983 [23]. Le point culminant du parc est "Ras El Braret" avec $1.787 \mathrm{~m}$ d'altitude. Le point le plus bas est à $858 \mathrm{~m}$, soit une altitude moyenne de 1.320 $\mathrm{m}$. Le climat est un facteur très important dans la répartition et la présence de la végétation. Celui de la région de Theniet El Had est de type méditerranéen tempéré. Le parc national reçoit en moyenne dans les 800 à $900 \mathrm{~mm}$ de pluie par an. La période hivernale est longue et rude, l'été est tempéré [23]. Le peuplement forestier représente les $3 / 4$ de la superficie totale du parc, le reste est à l'état de maquis. D'après [69], les inventaires floristiques menés ont permis de recenser 556 espèces végétales entre phanérogames (angiospermes, gymnospermes), et cryptogames (thallophytes, bryophytes et ptéridophytes) dont dix (10) endémiques algériennes. Il existe encore plusieurs espèces surtout herbacées qui restent à identifier et qui dénotent la grande richesse du parc ; Lamiaceae, Asteraceae, Caryophyllaceae, Fabaceae et Asteraceae.

Le mélange des espèces végétales présente aussi trois particularités notables, à savoir :

- Le mélange du cèdre de l'Atlas (Cedrus atlantica) et le chêne zeen (Quercus faginea);

- Le côtoiement du cèdre de l'Atlas et du pistachier de l'Atlas (Pistacia atlantica)

- Les peuplements à chêne liège (Quercus suber) s'y individualisent en occupant des étages altitudinaux supérieurs (plus de 1.500 m), un fait très rare dans le pourtour méditerranéen. 


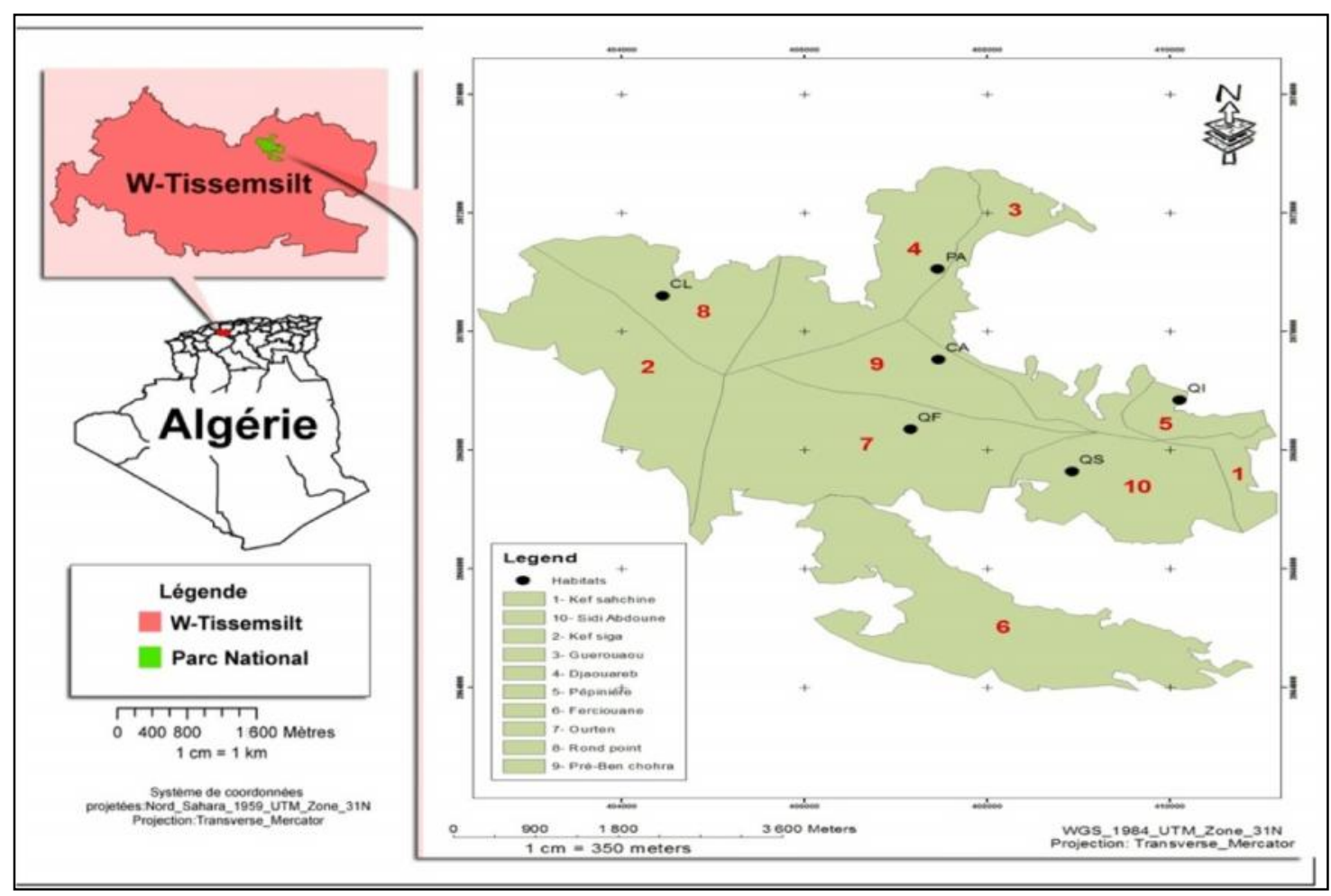

Fig.1. Carte de situation du parc national de Theniet El Had et localisation des différents types d'habitats [23].

1-Kaf Sachine, 2-Kaf-siga, 3-El Guerouaou, 4-Djouareb, 5-Pépinière, 6-Ferciouane, 7-Ourten, 8-Rond point, 9-Pré-Ben Chouhra, 10-Sidi Abdoune.

\section{METHODOLOGIE}

A l'aide d'un filet fauchoir à papillon, le piège lumineux et aérien, nous avons capturés les lépidoptères aux alentours des 06 stations choisies (Fig. 1). La clairière (pelouse), la cédraie, la yeusaie, la subéraie, la zénaie et la pinaie chacune occupe une superficie d'un hectare et regroupe 05 peuplements purs et une clairière. Le choix des sites a été distingué en fonction de leur facilité d'accès et par la diversité et le type de végétation qu'ils offraient. Nous avons effectués 84 sorties durant la période allant de mars 2015 jusqu'à octobre 2016 pour chaque station. Un nombre total de 851 individus a été récolté et un exemplaire de chaque espèce a été ramené au laboratoire pour identification.

\section{Méthodes d'analyses des données}

Nous avons utilisés des indices écologiques de compositions tels que la richesse totale (S) et moyenne $(\mathrm{Sm})$; l'abondance relative $(\mathrm{AR} \%)$ et la fréquence d'occurrence (FO \%) ainsi que l'indice écologique de structure de la diversité de Shannon (H') et l'équitabilité (E) pour exploiter nos résultats. 
La richesse des espèces est le nombre total d'espèces rencontrées au moins une fois au cours de $\mathrm{N}$ observations, et la richesse moyenne est le nombre moyen d'espèces rencontrées au cours de chaque évènement de comptage [56].

* L'abondance relative est le pourcentage d'individus d'une espèce donnée par rapport au nombre total des exemplaires. Elle est exprimée par la formule suivante: $\operatorname{AR}(\%)=($ ni / N) X 100 (ni: nombre d'individus d'espèces i; N: nombre total d'individus de toutes les espèces confondues) [12].

* D'après [13] la fréquence d'occurrence est calculée par la formule suivante : FO $\%=$ ni1 / N2 x 100 ; FO\%: Fréquence d'occurrence ; ni1 : Nombre de relevés contenant l'espèce i; N2 : Nombre total de relevés. Pour déterminer le nombre de classes de constance (N.c.), nous avons utilisé l'indice de Sturge [22]: N.c. $=1+(3,3 \log 10$ N3) N3 représente le nombre total des individus capturés grâce aux trois techniques utilisées.

* L'indice de la diversité de Shannon-Weaver [7] est calculé selon la formule suivante : $H^{\prime}=-\Sigma$ pi $\log 2$ pi (bits); H': Indice de diversité de Shannon-Weaver; pi : Probabilité de rencontrer l'espèce $\mathrm{i}$ obtenue par l'équation suivante : pi = ni / N ; ni : Nombre des individus de l'espèce i; $\mathrm{N}$ : Nombre total des individus de toutes les espèces échantillonnées.

* Selon [8], l'indice d'équitabilité ou d'équirépartition correspond au rapport de la diversité observée (H') à la diversité maximale (H' $\mathrm{E}=\mathrm{H}$ ' / H' max.; E : Indice d'équitabilité; H' : Indice de diversité de Shannon-Weaver ; H' max. : Diversité maximale, donnée par la formule suivante : H' max. $=\log 2 \mathrm{~S} ; \mathrm{S}$ : Richesse totale exprimée en nombre d'espèces.

* Parmi les méthodes statistiques employées pour exploiter les résultats l'analyse factorielle des correspondances (A.F.C.), nous avons utilisé le logiciel XLSTAT-Pro3DPlot7.01.

\section{RESULTATS}

La liste présentée ci-dessous est classée selon la systématique de [25], Les espèces indiquées en italiques sont suivies du nom de leur auteur et de la date de description des taxons, selon la même nomenclature (Tab.1).

\subsection{Liste des espèces}


Tableau 1. Inventaire globale des espèces de lépidoptères aux alentours du parc national de Theniet El Had durant 24 mois des années 2015 et 2016.

\begin{tabular}{|c|c|c|c|}
\hline Clade & Super familles & Familles & Espèces \\
\hline \multirow[t]{10}{*}{ Apoditrysia } & Alucitoidea & Alucitidae & Alucita hexadactyla (Linnaeus, 1758) \\
\hline & Pterophoroidea & Pterophoridae & Crombrugghia laetus (Zeller, 1847) \\
\hline & & & Emmelina monodactyla (Linnaeus, 1758) \\
\hline & Cossoidea & Cossidae & Cossus cossus (Linnaeus, 1758) \\
\hline & & Sesiidae & Chamaesphecia pechi (Staudinger, 1887) \\
\hline & & & Pyropteron leucomelaena (Zeller, 1847) \\
\hline & Zygaenoidea & Heterogynidae & Heterogynis penella (Hübner, 1819) \\
\hline & & Zygaenidae & Adscita mauretanica (Naufock, 1932) \\
\hline & & & Zygaena favonia (Freyer, 1844) \\
\hline & & & Zygaena zuleima Pierret, 1834 \\
\hline \multirow[t]{34}{*}{ Obtectomera } & Papilionoidea & Papilionidae & Iphiclides feisthamelii (Duponchel, 1832) \\
\hline & & & Zerynthia rumina (Linnaeus, 1758) \\
\hline & & Hesperiidae & Carcharodus tripolinus (Verity, 1925) \\
\hline & & & Pyrgus cinarae (Rambur, 1842) \\
\hline & & Pieridae & Anthocharis belia (Linnaeus, 1767) \\
\hline & & & Aporia crataegi (Linnaeus, 1758) \\
\hline & & & Colias croceus (Fourcroy, 1785) \\
\hline & & & Gonepteryx cleopatra (Linnaeus,1767) \\
\hline & & & Pieris brassicae (Linnaeus, 1758) \\
\hline & & & Pieris rapae (Linnaeus, 1758) \\
\hline & & & Pontia daplidice (Linnaeus, 1758) \\
\hline & & Lycaenidae & Callophrys avis (Chapman, 1909) \\
\hline & & & Lycaena phlaeas (Linnaeus, 1761) \\
\hline & & & Neozephyrus quercus (Linnaeus, 1758) \\
\hline & & & Polymmatus amandus (Schneider, 1792) \\
\hline & & & Polymmatus escheri (Hübner, 1823) \\
\hline & & Nymphalidae & $\begin{array}{l}\text { Argynnis pandora (Dennis et Schiffermüller, } \\
1775)\end{array}$ \\
\hline & & & Coenonympha pamphilus (Linnaeus, 1758) \\
\hline & & & Hipparchia ellena (Oberthür, 1893) \\
\hline & & & Hipparchia algirica (Oberthür, 1876) \\
\hline & & & Hyponephele lupina (Costa, 1836) \\
\hline & & & Lasiommata megera (Linnaeus, 1767) \\
\hline & & & Maniola jurtina (Linnaeus, 1758) \\
\hline & & & Melanargia galathea (Linnaeus, 1758) \\
\hline & & & Neohipparchia fatua (Freyer, 1844) \\
\hline & & & Neohipparchia statilinus (Hufnagel, 1766) \\
\hline & & & Nymphalis polychloros (Linnaeus, 1758) \\
\hline & & & Pararge aegeria (Linnaeus, 1758$)$ \\
\hline & & & Pyronia janiroides (Herrich-Schäffer,1851) \\
\hline & & & Vanessa atalanta (Linnaeus, 1758) \\
\hline & & & Vanessa cardui (Linnaeus, 1758) \\
\hline & Pyraloidea & Pyralidae & Aglossa pinguinalis (Linnaeus, 1758) \\
\hline & & & Plodia interpunctella (Hübner, 1813) \\
\hline & & & Pyralis farinalis (Linnaeus, 1758) \\
\hline
\end{tabular}




\begin{tabular}{|c|c|c|c|}
\hline \multirow{4}{*}{ Macroheterocera } & \multirow{4}{*}{ Bombycoidea } & \multirow{4}{*}{ Sphingidae } & Stemmatophora vulpecalis (Ragonot, 1891) \\
\hline & & & Synaphe interjunctalis (Guenée, 1849) \\
\hline & & & Hippotion celerio (Linnaeus, 1758) \\
\hline & & & Macroglossum stellatarum (Linnaeus, 1758) \\
\hline \multirow[t]{18}{*}{ Macroheterocera } & Geometroidea & Geometridae & Adactylotis gesticularia (Hübner, 1817) \\
\hline & & & Colotois pennaria (Hübner, 1823) \\
\hline & & & Idaea cervantaria (Millière, 1869) \\
\hline & & & Idaea ochrata (Scopoli, 1763) \\
\hline & & & Selidosema picturata (Rothschild, 1914) \\
\hline & Noctuoidea & Notodontidae & Thaumetopoea bonjeani (Powell, 1922) \\
\hline & & & $\begin{array}{l}\text { Thaumetopoea pityocampa (Denis \& } \\
\text { Schiffermüller, 1775) }\end{array}$ \\
\hline & & Erebidae & Apopestes spectrum maura (Warren, 1913) \\
\hline & & & Catocala ilia (Cramer, 1776) \\
\hline & & & Catocala delilah (Strecker, 1874) \\
\hline & & & Catocala nupta(Linnaeus, 1767) \\
\hline & & & Eublemma parva (Hübner, 1808) \\
\hline & & & Lymantria dispar (Linnaeus, 1758) \\
\hline & & & Notarctia proxima (Guérin-Méneville, 1844) \\
\hline & & Noctuidae & Autographa gamma (Linnaeus, 1758) \\
\hline & & & Dichonia aprilina (Linnaeus, 1758) \\
\hline & & & Episema scillae (Chrétien, 1888) \\
\hline & & & Euxoa rugifrons (Mabille, 1888) \\
\hline
\end{tabular}

Nous avons capturé au total 60 espèces réparties entre 3 clades, 9 superfamilles et 17 familles. Celle des Nymphalidae est représentée par le plus grand effectif en espèces. Les effectifs des espèces de lépidoptères selon les 6 types d'habitats durant les 24 mois d'étude sont regroupés dans le tableau 2.

Tableau 2. Effectifs des espèces de lépidoptères selon les 6 types d'habitats durant les 24 mois d'étude.

\begin{tabular}{lcccc}
\hline $\begin{array}{c}\text { Types } \\
\text { d'Habitats }\end{array}$ & Rhopalocères & Hétérocères & Sommes & $\begin{array}{c}\text { Pourcentage } \\
\text { d'effectifs\% }\end{array}$ \\
\hline Cédraie & 25 & 14 & 39 & 18.75 \\
Zénaie & 22 & 07 & 29 & 14.42 \\
Suberaie & 23 & 07 & 30 & 14.42 \\
Yeusaie & 22 & 11 & 33 & 15.87 \\
Pinaie & 16 & 06 & 22 & 10.58 \\
Clairière & 31 & 23 & 54 & 25.96 \\
(pelouse) & & & & 100 \\
\hline \multicolumn{1}{c}{ Totales } & 139 & 68 & 208 & \\
\hline
\end{tabular}

Le tableau 2 montre que l'effectif de la clairière (pelouse) est dominant en nombres d'espèces avec un total de 54 espèces et un pourcentage de 25,96\%. 39 espèces pour la cédraie, 33 
espèces pour la yeusaie, 30 espèces pour la subéraie, 29 espèces pour la zénaie et enfin 22 espèces pour la pinaie.

\subsection{Richesse totale $(\mathrm{S})$ et richesse moyenne $(\mathrm{Sm})$ selon les 6 types d'habitats}

La richesse totale $(S)$ varie d'un habitat à l'autre. Le nombre d'espèces exploitées à des stations varie entre 22 et 54 . La richesse la plus élevée est enregistrée pour la clairière avec 54 espèces. Suivi par la cédraie avec 39 espèces. Puis par la yeusaie avec 33 espèces, la subéraie avec 30 espèces. Ainsi que la zénaie avec 29 espèces et enfin la pinaie avec 22 espèces. La richesse moyenne varie de 0.92 à 2.25 (Tab. 3).

Tableau 3. La richesse totale $(\mathrm{S})$ et richesse moyenne $(\mathrm{Sm})$ et indices écologiques de structure des espèces de lépidoptères observées aux alentours des 6 types habitats dans le parc national de Theniet El Had durant 24 mois d'étude

\begin{tabular}{lccccc}
\hline \multicolumn{1}{c}{ Types d'Habitats } & S & Sm & H'(bits) & $\begin{array}{c}\text { H'max. } \\
\text { (bits) }\end{array}$ & E \\
\hline Cédraie & 39 & 1,63 & 2,58 & 3,32 & 0,78 \\
Zénaie & 29 & 1,21 & 2,21 & 2,81 & 0,79 \\
Suberaie & 30 & 1,25 & 2.12 & 2,81 & 0,76 \\
Yeusaie & 33 & 1,38 & 2.28 & 3.00 & 0,76 \\
Pinaie & 22 & 0,92 & 2.17 & 2.81 & 0.77 \\
Clairière (pelouse) & 54 & 2,25 & 2.73 & 4.00 & 0,68 \\
\hline
\end{tabular}

S: richesse totale; Sm: espèces moyenne la richesse; H: Shannon-Weaver indice de diversité; Hmax: diversité maximale; E: équirépartition.

\subsection{Abondance relative}

Abondance relative (AR\%) dans le parc de Theniet El Had note 851 individus recensés (Tab. 4). La clairière (pelouse) qui correspond au plus grand nombre d'effectifs avec 263 individus (A. R. $\%=30,9 \%$ ), suivis par la cédraie (188 ind., 22.9\%), la yeusaie (116 ind., 13.63\%), la subéraie (109 ind., 12.81\%) et la zénaie en dernier avec 104 individus (A. R. \% = 12.22\%). La plus faible valeur d'abondance des espèces a été enregistrée dans la pinaie avec 71 individus (A.R \% = 8.34\%) (Fig. 2). Les Nymphalidae sont placés en première position avec 32.90\% (280 individus). Suivi par la famille des Erebidae avec 149 individus (17.51\%). Puis par celle des Pieridae avec 147 individus (17.27\%). Après vient la famille des Lycaenidae avec 119 individus (13.98\%). Ensuite la famille des Notodontidae avec 58 individus (6.82\%). Concernant les autres familles leurs taux varient de $0.12 \%$ (1 individu) à $4.11 \%$ (35 individus) (Tab.4). 


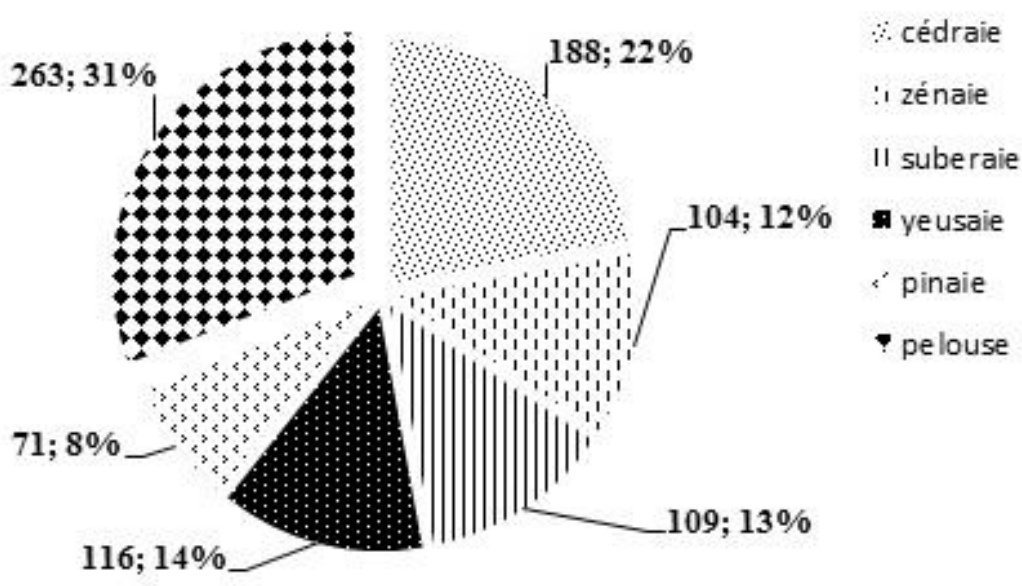

Fig.2. Abondance relative (AR\%) des espèces de lépidoptères selon les habitats

Tableau 4. Abondance relative (AR \%) et fréquence d'occurrence des familles d'espèces de lépidoptères recensés au niveau du parc national de Theniet El Had durant 24 mois des années 2015 et 2016.

\begin{tabular}{|c|c|c|c|c|c|c|}
\hline Clade & $\begin{array}{l}\text { Super } \\
\text { famille }\end{array}$ & Famille & ni & AR (\%) & $\mathrm{FO}(\%)$ & Catégories \\
\hline \multirow[t]{6}{*}{ Apoditrysia } & Alucitoidea & Alucitidae & 4 & 0,47 & 2.38 & Très rare \\
\hline & $\begin{array}{l}\text { Pterophoroid } \\
\text { ea }\end{array}$ & Pterophoridae & 8 & 0,94 & 8.33 & Très rare \\
\hline & \multirow[t]{2}{*}{ Cossoidea } & Cossidae & 1 & 0,12 & 1.19 & Très rare \\
\hline & & Sesiidae & 2 & 0,24 & 2.38 & Très rare \\
\hline & \multirow{2}{*}{ Zygaenoidea } & Heterogynidae & 1 & 0,12 & 1.19 & Très rare \\
\hline & & Zygaenidae & 9 & 1,06 & 5.95 & Très rare \\
\hline \multirow[t]{6}{*}{ Obtectomera } & \multirow[t]{5}{*}{ Papilionoidea } & Papilionidae & 1 & 0,12 & 1.19 & Très rare \\
\hline & & Hesperiidae & 2 & 0,24 & 2.38 & Très rare \\
\hline & & Pieridae & 147 & 17,27 & 41.67 & Accessoires \\
\hline & & Lycaenidae & 119 & 13,98 & 30.95 & Accidentelles \\
\hline & & Nymphalidae & 280 & 32,90 & 86.90 & $\begin{array}{l}\text { Fortement } \\
\text { constantes }\end{array}$ \\
\hline & Pyraloidea & Pyralidae & 35 & 4,11 & 15.48 & Rares \\
\hline \multirow{6}{*}{$\begin{array}{l}\text { Macroheteroc } \\
\text { era }\end{array}$} & Bombycoidea & Sphingidae & 17 & 2,00 & 2.38 & Très rare \\
\hline & $\begin{array}{l}\text { Geometroide } \\
\text { a }\end{array}$ & Geometridae & 3 & 0,35 & 3.57 & Très rare \\
\hline & & Notodontidae & 58 & 6,82 & 4.76 & Très rare \\
\hline & \multirow{3}{*}{ Noctuoidea } & Erebidae & 149 & 17,51 & 26.19 & Assez rare \\
\hline & & Noctuidae & 15 & 1,76 & 10.71 & Rare \\
\hline & & Totale & 851 & 100.00 & & \\
\hline
\end{tabular}




\subsection{Fréquence d'occurrences}

Fréquences d'occurrences globales (FO\%) selon les familles de lépidoptères $\mathrm{Au}$ niveau du parc national de Theniet El Had, 11 familles qui sont enregistrées les plus grands nombres de cas d'espèces appartenant à la classe très rare allant de $1.19 \%$ à $8.33 \% .2$ familles rares avec un taux de $10.15 \%$ et $15.48 \%$. Par contre une seule famille des Erebidae notée comme espèces assez rare avec 26.19\%. La classe fortement constante des espèces est enregistrée pour la famille des Nymphalidae (86.90\%). Effectivement elle est dominante au niveau de ses habitats. Egalement nous notons qu'une seule famille appartient aux classes des espèces accessoires les Pieridae avec $41.67 \%$ et suivie par la classe des espèces accidentelles des Lycaenidae (30.90\%) (Tab. 4).

\subsection{Indice de diversité}

L'indice de diversité (H') montre que la clairière (pelouse) semble être la plus diversifiée en termes d'espèces de papillons avec 2.73 bits suivies de la cédraie avec $\mathrm{H}^{\prime}=2.58$ bits. Puis vient la yeusaie avec 2.28 bits et Zénaie avec 2.21 bits. Ensuite la Pinaie et la subéraie avec 2.17 bits et 2.12 bits respectivement. La valeur d'équitabilité de tous les habitats est équilibrée en termes de nombres de papillons allant de 0.68 à 0.79 (Tab. 3).

\subsection{Analyse factorielle des correspondances}

Analyse des données par l'analyse factorielle des correspondances (AFC) Les familles de lépidoptères recensés dans différents habitats au niveau du parc national de Theniet El Had sont représentées dans la figure 4. Dans l'ensemble des habitats les familles de lépidoptères capturées contribuent à l'inertie totale avec $49.91 \%$ pour l'axe 1 et avec $24.66 \%$ pour l'axe 2 (Fig. 4). La somme des contributions des deux axes est de $74.57 \%$. Elle est supérieure à 50\%, donc le plan qui constitue ces deux axes est suffisant car il contient le maximum d'informations pour l'interprétation des résultats. Les habitats qui participent dans la formation de l'axe 1 sont surtout la pinaie avec 54,80\%, la pelouse avec $21,65 \%$ et la cédraie avec $17,55 \%$. Quant à la zénaie avec $2.08 \%$, la yeusaie avec $2.05 \%$ et la subéraie avec $1.86 \%$, elles interviennent moins. Pour la construction de l'axe 2 les stations qui contribuent le plus sont la yeusaie avec $74.77 \%$, la pelouse avec $14,00 \%$, la cédraie avec $9.73 \%$. Par contre celle de la pinaie intervient faiblement avec $1,21 \%$. Ainsi que la subéraie et la zénaie avec $0.18 \%$ et $0.11 \%$ respectivement. La yeusaie et la subéraie se situe dans le premier quadrant, cela signifie qu'il existe une association des espèces de papillons entre les deux habitats. La pinaie se situe dans le deuxième quadrant et la cédraie dans le troisième. Par contre la zénaie et la pelouse se situent dans le quatrième quadrant ce qui montre qu'il existe un rapprochement 
entre les compositions en espèces des papillons dans ces deux habitations du parc national de Theniet El Had. La famille qui intervient le plus dans la formation de l'axe 1, avec un pourcentage égal à 84,66\% est celle désignée par Notodontidae (Not). Pour ce qui est de l'axe 2 celle qui participe le plus à une contribution égale à $58.50 \%$ est la famille des Lycaenidae. Il existe 6 groupements particuliers les familles des papillons capturés aux niveaux des différents habitats qui retiennent l'attention. Ils sont signalés par G1, G2, G3, G4, G5 et G6. Le nuage de points $\mathrm{A}$ se situe près de l'intersection des axes 1 et 2 , et regroupe 6 familles qui sont omniprésentes. Ces familles sont représentées notamment par des Pterophoridae (Pte), les Pieridae (Pier), les Lycaenidae (Lyc), les Nymphalidae (Nym), les Pyralidae (Pyr) et Erebidae (Ere). Le groupement G2 concerne les familles mentionnées seulement à la clairière (pelouse) représentées surtout par les Cossidae (Cos), les Sesiidae (Ses), les Heterogynidae (Het), les Papilionidae (Pap), Sphingidae (Sph) et les Hesperiidae (Hes). Le nuage de points du groupe G3 contient une seule famille appart qui occupe les 4 habitats au même temps la cédraie, la pelouse, la zénaie et la subéraie, notamment les Noctuidae (Noc). Le nuage de points de G4 renferme une seule famille observée uniquement à la pinaie et la cédraie (ELM). Ce groupement comprend la famille de Notodontidae (Not). Pour ce qui est du G5, il contient une seule famille particulière à la yeusaie et la pelouse, représentés surtout par la famille des Alucitidae (Alu) (Fig. 3). La répartition des 2 familles sont regroupés dans le G6 qui comprennent les familles des Zygaenidae (Zyg) et des Geometridae (Geo) dans deux habitats différents : la cédraie et la pelouse. Cette répartition des familles dans différents habitats peut être expliquée avec les différences entre les essences floristiques qui se composent dans chaque habitat au sein de ce parc. 


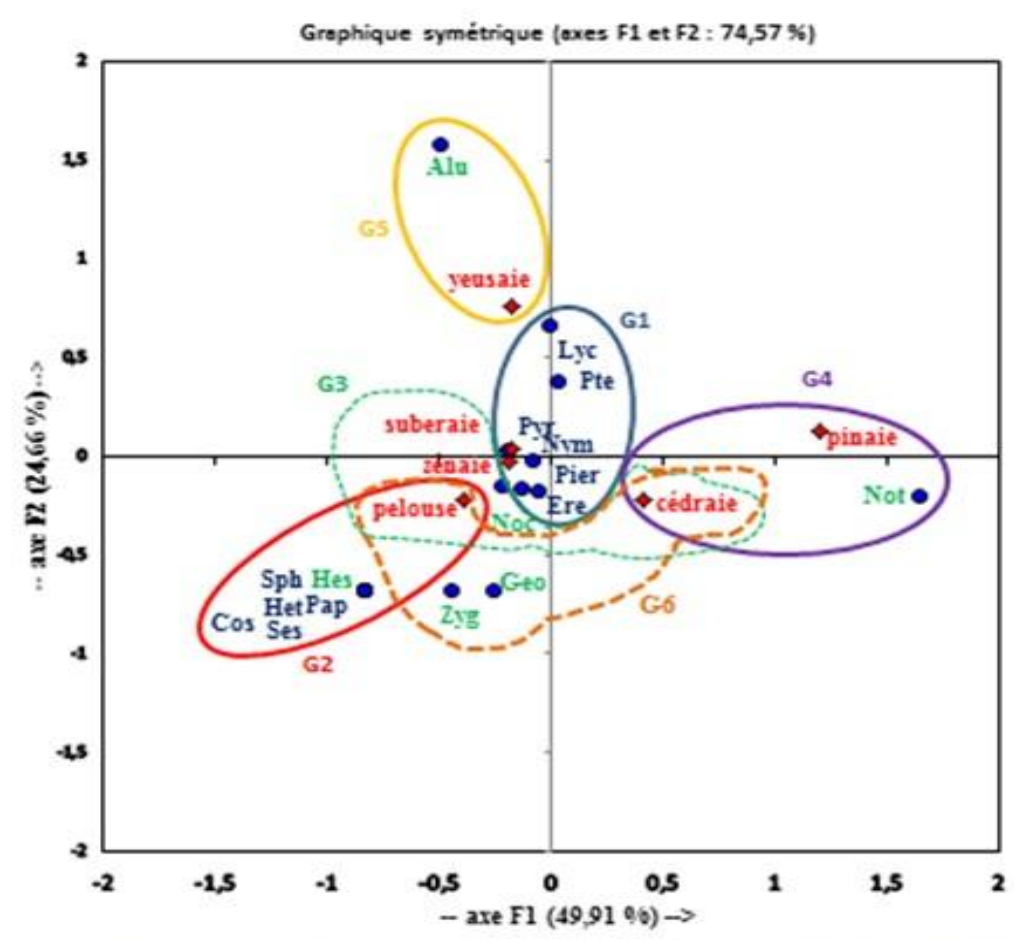

Fig.3. Répartition des familles de papillons entre les habitats d'étude, tel que déterminé par l'analyse factoriel de correspondance (AFC).

\section{DISCUSSIONS}

Les papillons ont la possibilité de se déplacer beaucoup plus facilement même si, en règle générale, ils se cantonnent aux milieux où la présence de la plante nourricière permettra le développement de la larve. Les Vanesses (Nymphalidae) par exemple, peuvent parcourir de grandes distances à la recherche du nectar des fleurs [4] mais aussi de miellat, d'exsudants de sève ou d'excréments pour puiser leur énergie. Ils se plaisent également à fréquenter des zones légèrement humides (flaques et les mares), aux bords desquelles ils trouvent des sels minéraux [68]. Dans cette étude, nous avons capturé au total 60 espèces réparties entre 3 clades, 9 superfamilles et 17 familles. Celle des Nymphalidae est représentée par le plus grand effectif en espèces. Le suivi des espèces de lépidoptères selon les 6 types d'habitats durant les 24 mois d'étude a permis de capturer un nombre d'espèces variant entre 22 et 54 . La richesse la plus élevée est enregistré pour la clairière (pelouse) avec 54 espèces. Suivi par la cédraie avec 39 espèces. Puis par la yeusaie avec 33 espèces, la subéraie avec 30 espèces. Ainsi que la zénaie avec 29 espèces et enfin la pinaie avec 22 espèces. La richesse moyenne varie de 0.92 à 2.25. Nos résultats confirmes à ceux de [53] qui ont enregistré un total de 2.276 individus représentant 22 espèces dans cinq paysages différents à l'ouest de la France, il s'agit d'un 
complexe mosaïque, une forêt-prairie, un champs de maïs et de grandes prés temporaires; un mosaïque de plantes cultivées (le maïs et le blé); un mosaïque de prairies à petite échelle et des parcelles de plantes cultivées ; un complexe d'anciennes prairies, un verger et champs en jachère. Alors que [28] a noté 32 espèces de lépidoptères réparties entre Rhopalocères et Hétérocères sur divers milieux constituant la zone naturelle du Marais du Curnic en Guissény (Bretagne), des étangs saumâtres proches du littoral, des friches humides ou sèches bordant ces plans d'eau, des prairies humides et mares située en arrière des précédents, des zones de prairies tourbeuses, de roselière et de saulaies situées au sud-ouest de la zone. Tandis que 202 espèces de papillons appartenant à 18 familles capturé par [63] dans deux localités de sud d'Algérie le Hoggar et le Tassili. Par contre [57] ont également signalé 22 espèces de papillons présentes dans les paysages agricoles de Mitidja en Algérie, comprenait les différents types de cultures qui y sont cultivées : verger d'agrumes, parcelle de maraichages, le verger de poiriers, le verger de pêchers et une parcelle de céréales. Ces différences enregistrées dans la diversité des espèces peuvent être expliquées par des périodes d'échantillonnage, qui sont liés à la présence de plantes nourrissantes. D'après [21], souligne qu'une plante nourrissante est parmi les facteurs clés dans le maintien des espèces d'insectes dans un habitat.

La diversité notée (H') dans notre station montre que la clairière (pelouse) semble être la plus diversifié en termes d'espèces de papillons avec 2.73 bits suivies de la cédraie avec $\mathrm{H}^{\prime}=2.58$ bits. Puis vient la yeusaie avec 2.28 bits et la zénaie avec 2.21 bits. Ensuite la pinaie et la subéraie avec 2.17 bits et 2.12 bits respectivement. La valeur d'équitabilité de tous les habitats est équilibrée en termes de nombres de papillons allant de 0.68 à 0.79 (Tab. 3). Effectivement la diversité est utilisée pour comparer la richesse des espèces dans deux biocénoses, en particulier lorsque le nombre des individus récoltés dans chacun d'entre eux est très différent. La diversité est conditionnée par deux facteurs, à savoir la stabilité de l'environnement et des facteurs climatiques [12]. Plusieurs habitats représentent des environnements stables avec des conditions aussi favorables pour les papillons. Dans notre présente étude la famille des Nymphalidae, reste la plus riche en espèces de nombre de 15 dans tous les types d'habitats. Suivi par la famille des Erebidae, des Pieridae et des Pterophoridae variant de 2 à 7 espèces (Tab.1 ; Fig.4). Cette présence des familles de papillons dans les 6 habitats est due à la richesse floristique qui bordent le parc national de Theniet El Had.

La clairière (pelouse) est l'une des habitats les plus riches soit par le nombre d'individus de papillon (tableau 3) ou par les plantes et fleurs où plusieurs sources d'eaux au niveau du parc 
dont certaines sont captées et utilisées pour les besoins de la faune. Citons la source Ain Touilla, la source Ain Harhara, la source Djedj El Ma, la source de Toursout et retenue collinaire au Sud du Sidi Abdoun. Cette dernière est dominée par la strate herbacée riche en Astéracées telle que Bellis perennis, Bellis annua, Eryngium campestre, Paronychia argentea, Salix alba. Les orchidacées comme Orchis simia, Orchis papilionacea, Orchis italica, Ophrys fuciflora, Orphys tenthredinifera. Les Fabacées : avec Calicotum spinosa, Vicia sicula. Les Apiacées présentés par Ferula communis. Les Liliacées : Bellevalia romana, Narcissus cantabrica. Les Iridacées: Romulea bulbocodium. Les Lamiacées avec l'espèce Phlomis crinita. Les Violaceae : Viola munbyana, Les Geraniaceae : Geranim atlanticum et enfin les Brassicacées avec Alliaria officinalis [5]. A l'exposition sud de la clairière; nous remarquons la réduction du tapis végétal en matière de diversité forestière ; cela est due essentiellement au pâturage traduit par la présence des animaux domestiques et l'installation d'espèces opportunistes qui favorise le pâturage tels que Calendula arvensis, Anacyclus clavatus, Marrubium vulgare, Malva sylvestris, Plantago lagopus, Rumex bucephalophorus, Reseda alba [44]. Nous remarquons l'absence d'une seule famille, celle des Notodontidae, dans la clairière. D'après [47], les milieux moins perturbés contiennent une faune plus riche et plus abondante, plusieurs espèces ne se trouvent qu'en forêt ou sur les clairières. Le pâturage constitue un phénomène étalé dans le temps, progressif, et qui se double d'un piétinement plus ou moins intense, conduisent rapidement à la dégradation du couvert végétal et du sol contribuant à l'appauvrissement généralisé des communautés animales et végétales [37].

Les chênes verts occupent une superficie de 1000 ha dans l'habitat de la yeusaie [70]. Huit familles de papillons sont notées dans la yeusaie dont la famille la mieux représentée est celle des Lycaenidae (42 individus), suivi par les Nymphalidae (37individus) et Pieridae (16 individus) (Tab. 3). Parmi les espèces de papillons les plus abondantes sont : Neozephyrus quercus avec 27 individus, Pieris rapae et Hipparchia algirica avec 09 individus chacun, Maniola jurtina et Argynnis pandora avec 06 individus chacun (Tab. 1). Les feuilles du chêne vert sont les plus recherchées par les lépidoptères. Parmi lesquelles; la Thécla du chêne Neozephyrus quercus [4], indique que le Chêne vert joue un rôle important comme zone de refuge. On s'aperçoit que lors des fortes chaleurs un grand nombre de papillons se tiennent à l'ombre du feuillage des Chênes vert.

La cédraie occupe le versant Nord, le recouvrement des peuplements est très important (7080\%). Dans cette strate, la densité est très élevée 400 pieds /ha); la superficie globale est estimée à 1000 ha [70]. 10 familles sont capturées aux niveaux de cette habitat dont la famille 
la mieux représentée est celle des Nymphalidae avec 53 individus, suivi par les Erebidae (41 individus), les Pieridae (35 individus), les Notodontidae (32 individus). Puis viennent les Lycaenidae avec 12 individus et les autres familles sont faiblement présentes allant de 1 à 4 individus (Tab. 3). L'espèce la Mariée Catocala nupta est la plus répandue avec un taux de 6\% (18 individus). [54] mentionnent que la mariée au repos et en cachant ses ailes postérieures est très bien camouflée sur l'écorce des arbres ; dérangée elle s'enfuit en dévoilant ses ailes rouges et s'abat au sol pour échapper au prédateur éventuel. Elle colonise les forêts de feuillus humides, les bords des cours d'eau, étangs, forêts alluviales. Suivi par Thaumetopoea bonjeani et Catocala ilia avec 17 individus chacun, Thaumetopoea pityocampa et Pieris brassicae avec 15 individus chacun.

La cédraie de Theniet El Had connait un dépérissement depuis plusieurs années suites à l'interaction de plusieurs facteurs destructeurs, entre autre les insectes, plus spécifiquement les lépidoptères défoliateurs. La processionnaire du cèdre Thaumetopoea bonjeani et Thaumetopoea pityocampa [6]. D'après [40] qui a travaillé en 2009 sur le dépérissement dans le parc national de Theniet El Had (Alger) représente un taux le plus important, est le canton Guerouaou 26.24\% suivi par le canton Pré Benchouhra 18.77\%. L'existence des arbres réceptifs et affaiblie attirent les espèces agressives et les ravageurs redoutables. Selon [29]; [30] et [42], Thaumetopoea bonjeani est présente dans toutes les cédraies algériennes : Aurès, Djurdjura, Babors, Theniet El Had, Boutaleb et Chréa. Ces auteurs n'ont cité qu'une espèce : T. pityocampa. En juin 1982, une nouvelle chenille processionnaire de cèdre Thaumetopoea bonjeani causant des dégâts importants a été signalée dans la cédraie du Belezma (massif des Aurès) avec une forte présence dans ce massif, dont elle avait provoqué une grave défoliation plus de 80\% sur plus de 500 ha en 1984 [29]. Or qu'en Bulgarie, [3], [1] et [55] ont notés la présence des deux Thaumetopoea bonjeani et Thaumetopoea pityocampa sur la cédraie. Tandis que [27] a signalé la présence d'une autre espèce sur les cônes du cèdre du P.N.T.E.H, Dioryctria peltieri (de Joannis, 1908), elle n'est pas citée dans notre inventaire.

La subéraie du parc national de Theniet El Had se localise dans le versant sud, plus particulièrement dans les cantons Ourten et Sidi Abdoune [70]. Sa superficie globale est estimée à 680 ha. Cet habitat est caractérisé par la présence des peuplements à chêne liège, Quercus suber, qui occupent des étages altitudinaux supérieurs (plus de $1500 \mathrm{~m}$ ), qui sont très rare dans le pourtour méditerranéen. Les familles les plus abondantes dans la subéraie sont de nombre de 7 citons les Nymphalidae (38 individus), les Erebidae (35 individus), les Pieridae (16 individus), les Lycaenidae (15 individus), les Pterophoridae et les Noctuidae 1 individu 
chacun dont les espèces de papillons présentes dans la subéraie sont : Lymantria dispar avec 15 individus, Catocala nupta (09 individus), Maniola jurtina (08 individus), Catocala delilah et Hipparchia algirica avec 07 individus chacun. Nous remarquons la dominance du Bombyx disparate (Lymantria dispar) dans la subéraie. Cette espèce est signalée par [3].Les défoliateurs sont les principaux ravageurs du chêne-liège. Les Notodontidae et les Erebidae, avec plus précisément Lymantria dispar, sont les familles qui regroupent la majorité des espèces défoliateurs inventoriées et qui sont responsables pour la majorité des dégâts observés sur les arbres forestiers. D'après [31], les Thérophytes sont les plus représentées dans le parc national de Theniet El Had. En effet, un nombre élevé de thérophytes indique une forte perturbation du milieu par l'activité anthropozoïque, notamment le pâturage [11]. Le Bombyx disparate est le lépidoptère qui provoque les dégâts. Parmi les principaux phyllophages qui vivent au dépence du feuillage du chêne-liège autres que Lymantria dispar sont Orgyia trigotephras, Catocala nymphagoga, Tortrix viridana et d'autres espèces de la famille des Geometridae [10]. D'après [67], lors de la phase de culmination, la densité des chenilles devient considérable et les surfaces des forêts défoliées peuvent atteindre des dizaines de milliers d'hectares. Alors que [10] notent que certaines espèces sont capables d'attaquer l'arbre sur pied et sont dits Primaires. Parmi lesquelles ; le Gâte-bois, Cossus cossus. Nous avons trouvé un seul individu de ce dernier, au niveau d'une clairière. La zénaie, nous la trouvons dans quelques stations peu étendues dont le 1/3 est répandu en forme de futaie en exposition Nord, les 2/3 restent en exposition Sud au stade de gaulis ou perchis [70]. Nous avons recensés 7 familles dont la famille la plus répondus est celle des Erebidae avec 35 individus, suivi par les Nymphalidae (34 individus), les Pieridae (15 individus) et les Lycaenidae (11 individus). Les autres familles sont faiblement représentées avec 1 à 6 indivius (Tab. 3). Les espèces de papillons les plus abondantes dans la zénaie sont : Catocala ilia avec 14 individus, Catocala nupta (13 individus), Catocala delilah (08 individus), Maniola jurtina et Nymphalis polychloros 07 individus chacun. La mariée Catocala ilia est l'espèce la plus abondante dans la zénaie par rapport aux autre 06 types d'habitats étudiés. Les défoliations du chêne zeen sont dus à la Tordeuse verte; Tortrix viridana, ou à des Phalènes Géométrides : Cheimatobie; Operophthera brumata et Hibernie ; Erannis defoliaria, celles en mai et début du mois de juin sont dus à la Processionnaire du Chêne, alors que ceux de la fin du printemps, les dégâts sont causés par Bombyx disparate (Lymantria dispar) d'après [48]. Concernant la pinède, elle s'étend sur une superficie de 800 ha, caractérisé par de vielles futaies, occupant principalement les basses altitudes du versant Nord du canton 
Guerouaou [70]. Sur le pin d'Alep, nous avons recensé 7 familles, celles des Notodontidae qui dominent avec 26 individus, suivi par celles des Nymphalidae (20 individus), les Lycaenidae (14 individu). Puis les autres familles sont moins fréquent allant de 1à6 individus. Parmi les 22 espèces capturées dans ce type d'habitat et les plus abondantes citons Thaumetopoea bonjeani et Thaumetopoea pityocampa 13 individus chacun et Maniola jurtina (08 individus). La chenille Processionnaire du pin, Thaumetopoea pityocampa est l'un des ravageurs les plus redoutables du pin d'Alep. Les défoliations occasionnées par cet insecte affaiblissent les arbres attaqués et provoquent des pertes de croissance et de production considérables [14]; [35]; [61] \& [36]. En Algérie, on cite les travaux sur les défoliateurs du pin d'Alep de [71] qui a inventorié une entomofaune importante du pin d'Alep dans la forêt de Bainem (Alger) répartie entre les Coléoptères et les Lépidoptères. Celle de [41] a estimé les dégâts provoqués par Rhyacionia buoliana en Algérie et enfin [46] a estimé les dégâts de la Processionnaire du pin au niveau de la forêt de Nador (Tiaret). La présence des espèces de lépidoptères varie selon la structure du végétal considéré.

\section{CONCLUSIONS}

Cet inventaire des lépidoptères n'est évidemment qu'un aperçu de la faune des papillons de ce parc qui possède une grande richesse tant de la faune que de la flore. Les lépidoptères ainsi recensés présentent un grand nombre d'espèces citées pour la première fois dans le parc national de Theniet El Had. Parmi les 60 espèces de papillons capturés, les 22 espèces suivantes étaient déjà connues du parc : Thaumetopoea pityocampa, Thaumetopoea bonjeani, Lymantria dispar, Autographa gamma, Aporia crataegi, Colias croceus, Pieris rapae, Pieris brassicae, Pontia daplidice ,Anthocharis belia, Maniola jurtina, Hipparchia aristaeus, Melanargia galathea, Nymphalis polychloros, Argynnis pandora, Vanessa cardui, Neohipparchia statilinus, Coenonympha pamphilus, Vanessa atalanta, Zerynthia rumina, Lycaena phlaeas, Neozephyrus quercus et les 38 espèces de papillons qui restent sont considérées comme des espèces qui n'ont jamais été signalées au niveau de notre zone d'étude (Tab. 1).

La clairière est la plus peuplée en lépidoptères avec 54 espèces, la cédraie héberge 39 espèces suivi de la yeusaie avec 33 espèces, la subéraie et zénaie ne comportent que 30 et 29 espèces respectivement. La pinaie ne compte que 22 espèces. 
Parmi les différentes familles de la faune lépidoptérique les Nymphalidae sont les plus diversifiés en espèces (15 espèces avec 73 apparitions) et présentent le plus grand nombre d'individus (280) dans tous les habitats étudiés.

Les Notodontidae et les Erebidae sont les familles qui regroupent la majorité des espèces défoliatrices inventoriées (principalement Thaumetopoea bonjeani (30), Thaumetopoea pityocampa (28) et Lymantria dispar (20). Ils sont responsables pour la majorité des dégâts observés sur les arbres forestiers.

En effet, ce travail est une approche préliminaire ayant pour objectif principal d'établir une liste de la faune des lépidoptères du P.N.T.E.H.

Les six types d'habitat étudiés ne sont pas représentatifs dans tout le massif de Theniet El $\mathrm{Had}$, et il serait donc souhaitable de recenser la lépidoptérofaune dans d'autres habitats pour dresser une liste plus ou moins complète des principales espèces de cette réserve naturelle.

Pour terminer, nous encourageons donc le maintien de tels programmes d'inventaires naturalistes, particulièrement entomologiques, et bien sûr la prise en compte des lépidoptères dans les futures démarches en fonction des différents habitats.

\section{REFERENCES}

[1] Abdelhamid D. Contribution a l'étude des insectes du Cèdre de l'Atlas (c.a.m.1844) dans la Cédraie de T-E-H. Thèse Ingénieur, Université des sciences et technologie de BLIDA, Institut d'Agronomie, 68 p, 1992.

[2] Abdelhamid D. Etude bioécologique de l'entomofaune du Cèdre de l'Atlas, dans la Cédraie de T-E-H. Thèse Magister, Institut National Agronomique, Alger, 106 p, 1999.

[3] Anonyme. Etude et projets pour la mise en valeur des terres, aménagement des forêts et des parcs dans le massif de l'Ouarsenis. Lescomplekt Engineering Fiches descriptives ,1984, 19- 69 p. + annexes.

[4] Bachelard P., Morel D. Inventaire des Lépidoptères de la Réserve naturelle des Coussouls de Crau (Bouches-du-Rhône). Réalisé à la demande du Conservatoire - Etudes des Ecosystèmes de Provence, 2008,54 p.

[5] Belkaid B. Etude phytoécologie et possibilité d'amélioration dans la cédraie du Parc National de Théniet El Had. Thèse Ingénieur, Institut de Technologie Agricole, Mostaganem, $46 \mathrm{p}, 1988$.

[6] Bentouati A. La situation du cèdre de l'Atlas dans les Aurès (Algérie). Forêt méditerranéenne, 2, 2008,203-208. 
[7] Blondel J., Ferry C., Frochot B. Avifaune et végétation, essai d'analyse de la diversité. Alauda, 41 (1-2), 1973, 63-84.

[8] Blondel J. Biogéographie et écologie. Editions Masson, Paris, 1979, 173 p.

[9] Chadouli F. Contribution à l'inventaire de l'entomofaune en fonction de quatre ranches altitudinale de versants sud du parc national de T-E-H. Thèse Ingénieur, Université Ibn Khaldoun, Tiaret, 64 p, 2005.

[10] Chakali G., Ghelem M. Etat sanitaire des subéraies en Algérie. Annale de la recherche forestière au Maroc, 39, 2008, 93-99.

[11] Dahmani M. Diversité biologique et phytogéographique de chênaies vertes d'Algérie. Ecologia Mediteranea, 22, 1996,19-38.

[12] Dajoz R. Précis d'écologie. Editions Dunod, Paris, 1970, 357 p.

[13] Dajoz R. Précis d'écologie. Editions Gauthier-Villars, Paris, 1982,503 p.

[14] Demolin G. \& Rive. La processionnaire du pin en Tunisie. Annales de L'INRF de Tunisie, 1(1), 1968,1-19.

[15] De Prins W. Systematische Naamlijst van de Belgische Lepidoptera. Entomobrochure, 4, $1983,1-57$

[16] De Prins W. Scrobipalpa proclivella (Lepidoptera: Gelechiidae), a species new to Belgium. Phegea 36 (2), 2008, 57-58.

[17] De Prins W. Catalogue of the Lepidoptera of Belgium. Entomobrochure, 9, 2016, 1-279.

[18] De Prins W., De Prins J. Metalampra italica (Lepidoptera: Oecophoridae), also in Belgium. Phegea, 42 (2), 2014, 26-28.

[19] De Prins W., Steeman Ch., Sierens T. Interessante waarnemingen van Lepidoptera in België in 2014 (Lepidoptera). Phegea, 43 (4), 2015, 98-103.

[20] De Prins W., Mazzei P. Some faunistic notes on selected moth species (Lepidoptera) from the Seychelles. Phelsuma, 24, 2016, 21-34.

[21] Deschamps-Cottin M., Descimon H., Roux M. Valeur trophique des plantes nourricières et préférence de ponte chez Parnassius apollo L. (Lepidoptera, Papilionidae). Comptes Rendus de l'Académie des Sciences - Series III - Sciences de la Vie, 320 (5), 1997, 399-406. [22] Diomande D., Gourene G., Tito de Morais L. Stratégies alimentaires de Synodontis bastiani (Siluriformes: Mochokidae) dans le complexe fluvio-lacustre de la Bia, Côte d'Ivoire. Cybium, 25 (1), 2001, 7-21.

[23] D.P.N.T.H. Direction du Parc National de Théniet El Had (Département des resources naturelles). Présentation sur le Parc National De Theniet El Had, 2016, 14 p. 
[24] Efetov K., Hofmann A., Tarmann G. Application of two molecular approaches (use of sex attractants and DNA barcoding) allowed to rediscover Zygaenoprocris eberti (Alberti, 1968) (Lepidoptera, Zygaenidae, Procridinae), hitherto known only from the female holotype. Nota Lepidopterologica 37 (2), 2014, 151-160.

[25] Nieukerken E.J. van., Kaila L., Kitching I.J. \& al. Order Lepidoptera Linnaeus, 1758. In: Zhang Z-Q ed. Animal biodiversity: An outline of higher-level classification and survey of taxonomic richness. Zootaxa, 3148, 2011, 212-221.

[26] Faure É. Suivi de milieux ouverts dans le parc naturel régional du Luberon par des papillons de jour (Rhopalocères) bioindicateurs. Courrier scientifique du parc naturel régional du Luberon, n $8-2007,2007,86-101$.

[27] Fendil A. Etude des insectes liés aux cônes du cèdre de l'Atlas (Cedrus Atlantica.M) dans le parc national de Theniet el Had, Thèse Ingénieur, Université Ibn Khaldoun, Tiaret p. 67, 2007.

[28] Fouillet, P. Etude entomologique du Marais du curnic en Guissény (Finistère). Analyse des richesses des différents biotopes et propositions de mesures de gestion conservatoire favorables aux invertébrés. Etudes Faunistiques et Ecologiques, 1998, 6-30.

[29] Gachi M., Khémici M., Zamoum M. Note sur la présence en Algérie de la processionnaire du cèdre T. bonjeani Powell \{Lepidoptera thaumetopoeidaé). Annales de la Recherche Forestière en Algérie, 1, 1986, 53-63.

[30] Gachi M. Note sur la présence en Algérie de la processionnaire du Cèdre: Thaumetopoea bonjeani Powell (Lepidoptera ; Thaumetopoeidae). Annales de la Recherche Forestière en Algérie, 27, 1994, 527-537.

[31] Habib N. Contribution à l'élaboration d'une liste rouge de la flore vasculaire du Parc National de Théniet El Had (W. Tissemsilt). Thèse Ingénieur, Université Ibn Khaldoun,Tiaret, $51 \mathrm{p}, 2013$.

[32] Hausmann A. Thera britannica (Turner, 1925) (=Thera albonigrata Gornik) in Südbayern (Lep., Geom.). Nachrichtenblatt der Bayerischen Entomologen, 37 (4), 1988, 101103.

[33] Hausmann A. Faszination Biodiversität. In Herzog, E. \& H.-C. Bauer (2011): Blickpunkt: Darwin. Books on Demand, Salzburg, 211 p. [chapter pagination, 2011, 11-31]. [34] Hausmann A. Jagd nach urweltlichen Geometriden in Südafrika. Nachrichtenblatt der Bayerischen Entomologen, 64 (1/2), 2015, 62-63. 
[35] Hervouet L. Mesure des pertes de croissance radiale sur quelques espèces de Pinus dues à deux défoliateurs forestiers. I - Cas de la processionnaire du Pin en région méditerranéenne. Annales des Sciences forestières, 43 (2), 1986, 239-262.

[36] Jacquet J.-S. Impacts des défoliations de la processionnaire du pin (Thaumetopoea pityocampa) sur la croissance du pin maritime (Pinus pinaster). Thèse doctorat, Université de Bordeaux, 2012, 140 p.

[37] Jaulin S., Baillet Y. Identification et suivi des peuplements de Lépidoptères et d'Orthoptères sur l'ENS du Col du Coq - Pravouta. Rapport d'étude de l'OPIE-LR, Perpignan, 2007, 107 p.

[38] Joaquín Baixeras A. Book Review: Eucosma Hübner of the Contiguous United States and Canada (Lepidoptera: Tortricidae: Eucosmini). Nota Lepidopterologica, 38 (2), 2015, $157-158$.

[39] Jérémy D., Daniel V., Fabien V. Expérience territoires de conciliation Homme-Nature. Les Hétérocères ou «Papillons de nuit ». Groupe Associatif Estuaire, Paris, 2014, 2 p.

[40] Kacha S. Contribution à l'étude de quelques facteurs causaux du dépérissement du cèdre de l'Atlas (Cedrus atlantica Manetti) dans le parc national de Theniet el Had. Thèse d'Ingénieur, Université Ibn Khaldoun, Tiaret, 106 p, 2009.

[41] Kerris T. La tordeuse des pousses de pin (Rhyacionia buoliana Schiff.), répartition, dégâts et lutte en Algérie. Séminaire international sur les techniques de luttes contre la désertification, Alesco, Bou-Sâada, 1987, 1-25.

[42] Khemici M. Protection des cédraies en Algérie : Inventaire des insectes ravageurs et réseaux d'avertissement et de lutte. In : Workshop on Assessment of the scale of insect infestation in cedar forest in Lebanon and the Mediterranean region. University of Beirut, Liban, 2001,10-18.

[43] Lafranchis T. Les papillons de jour de France, Belgique et Luxembourg et leurs chenilles. Collection Parthénope, éditions Biotope, Mèze, 2000, 448 p.

[44] Letreuch B.A., Medjahdi B., Letreuch B.N. et al. Diversité floristique des subéraies du parc national de Tlemcen (Algérie). Acta botanica malacitana, 34, 2009, 78-89.

[45] Li H.H. The Gelechiidae of China [I] (Lepidoptera: Gelechioidea). Nankai University Press, Tianjin, 2002, 538 p.

[46] Maatoug M. Bio-écologie de la processionnaire du pin : Thaumetopoea pityocampa (Lep-Thaum) et estimation des dégâts dans la forêt de Nador (Tiaret). Thèse Ingénieur Forestier, Université de Tlemcen, 122 p, 1992. 
[47] Manil L., Lerch A., Edelist C. et al. Suivi temporel des Rhopalocères de France (STERF). Association des Lépidoptéristes de France (ALF) ; UMR 5173, Département Écologie et Gestion de la Biodiversité, Muséum national d'Histoire naturelle (MNHN), Paris. 2009, 41 p.

[48] Malphettes C.B. Les défoliateurs du chêne. Centre national du machinisme agricole, du génie rural, des eaux et des forêts INRA, 1990, 191-197.

[49] Martiré D., Merlier F., Turlin B. Guide des plus beaux papillons et leurs fleurs favorites. Editions Belin, Paris, 2016, 383 p.

[50] Neggaz B. Contribution à l'étude de l'entomofaune du chêne liège (Quercus suber) dans le parc national de T-E-H. Thèse Ingénieur, Université de Ibn Khaldoun, Tiaret, 66 p, 2005. [51] Oberthür C. Faunes entomologiques descriptions d'insectes nouveaux ou peu connus. Etude sur la faune des lépidoptères d'Algérie. Etudes d'Entomologie, pls I-IV., 1876, 1, 1-74. [52] Oberthür C. Faune des lépidoptères d'Algérie. Etudes de Lépidoptérologie comparée, X, 1915, 07-195.

[53] Ouin A., Burel F. Influence of herbaceous elements on butterfly diversity in hedgerow agricultural lLandscapes. Agriculture, Ecosystems and Environment, 1915,93, 2002, 45-53. [54] Perette L.N., Spill F., Rauch M. Les Papillons de la Réserve de la Biosphère des Vosges du Nord. Eguelshardt, Cicogna, 324 p. [chapter pagination, 2009, 209-210].

[55] Rahim N. Bio-écologie de la processionnaire du cèdre Thaumetopoea bonjeani Powell (Lepidoptera, Notodontidae) dans le Djurdjur. Thèse de Doctorat. Institut National Agronomique, Alger, 113 p, 2015.

[56] Ramade F. Eléments d'écologie. Ecologie fondamentale. Editions McGraw-Hill, Paris, 1984, 397 p.

[57] Remini L., Moulaï R. La diversité et la structure des populations de papillons dans les agro-écosystèmes de Mitidja (Algérie). Zoology and Ecology, 2015, 11 p.

[58] Rothschild L., Hartert E., Jordan K. Algerian lepidoptera. Explanation of figures on plate. Novitates Zoologicae, XXVII, 1920,544 p.

[59] Rungs E. Catalogue raisonné des lépidoptères du Maroc, Inventaire faunistique et observations écologiques. Institut scientifique, Rabat, Tome I (1979), 1-222, Tome II (1981), 1979-1981, 223-583.

[60] Senouci F. Initiation à l'inventaire de l'entomofaune de Cedrus atlantica (Cèdre de l'Atlas) et sa relation avec le dépérissement dans le parc national de T-E-H. Thèse Ingénieur, Université de Ibn Khaldoun, Tiaret, 64 p, 2006. 
[61] Sghaier T., Khouja M.L., Ben Jamaa M.H. Effet de la hauteur des arbres sur le comportement des provenances de pin d'Alep vis a vis des attaques de la processionnaire. Institut National de Recherches en Génie Rural Eaux et Forets, Tunis, 1999, 21-31 p.

[62] Soures B. Contribution à l'étude des Lépidoptères de la Tunisie. Biologie des espèces nuisibles ou susceptibles de le devenir. Publication Tunisie, 1 v, 1948, 211 p.

[63] Speidel W., Hassler M. Die Schmetterlingsfauna der südlichen algerischen Sahara und ihrer Hochgebirge Hoggar und Tassili n'Ajjer (Lepidoptera). Nachrichten des entomologischen Vereins Apollo, Supplement, 1989, 1-156.

[64] Tarmann G.M. The decline of Zygaenidae in the valleys of the Alps during the last 100 years. Abstracts of the XV International Symposium on Zygaenidae, Mals, 2016, 11-18 September 2016: 38 .

[65] Tolman T., Lewington R. Guide des Papillons d'Europe et d'Afrique du Nord. Editions Delachaux \& Niestlé, Neuchâtel-Paris, 1999, 320 p.

[66] Tolman T., Lewington R. Afrique du nord. Guide des papillons d'Europe et d'Afrique du nord. Editions Delachaux et Niestlé, 2014,384 p.

[67] Villemant C. Le Bombyx disparate en corse. Insectes, 3, 2003, 1-10.

[68] Violaine F. Plein phare sur nos papillons forestiers. Volets papillons de jour et libellules. Groupe de Travail "Lycaena". Echo des Réserves, 2006, 8-11, 2-4.

[69] Yahi N., Vela E., Benhouhou S. et al. Identifying Important Plants Areas (Key Biodiversity Areas for Plants) in northern Algeria. Journal of Threatened Taxa, 2012,13 p.

[70] Zedek M. Contribution à l'étude de la productivité du Cedrus atlantica Manetti. (Cèdre de l'Atlas) dans le parc national de Theniet el Had. Thèse Magister, Institut National Agronomique, Alger, 175 p + annexe, 1993.

[71] Zemmouri F. Contribution à l'étude de l'inventaire de l'entomofaune de Pinus halepensis Mill, Pinus pinaster L., Pinus radiata D. dans la forêt de Bainem (Alger). Thèse Ingénieur, Institut National Agronomique, Alger, 54 p, 1991. 


\section{LA RICHESSE ET LA DIVERSITE DES POPULATIONS DE LEPIDOPTERES DANS DIFFERENTS HABITATS DU PARC NATIONAL THENIET EL HAD (ALGERIE)}

\section{RESUME}

La diversité des espèces de lépidoptères étudiés au niveau du Parc National de Theniet El Had (P.N.T.E.H.) durant 24 mois des années 2015 et 2016 ; dans différents habitats totalise 851 individus de lépidoptères, appartenant à 17 familles, 09 superfamilles et 60 espèces. Parmi ces dernières 31 Rhopalocères et 35 Hétérocères ont été inventoriées. La clairière (pelouse) est la plus riche en papillons avec une richesse de 54 espèces. Suivi par celle de la cédraie avec 39 espèces, la yeusaie avec 33epèces, la subéraie avec 30 espèces, la zénaie 29 espèces, et enfin la pinaie avec seulement 22 espèces recensées. La famille des Nymphalidae est la plus dominante dans le parc avec un taux de 32.48\%. L'indice de diversité (H' et H'max.) et l'équitabilité (E) calculé au niveau des 6 types d'habitats est H'=2,74 bits, H'max.= 4,09 bits et $\mathrm{E}=0,67$ cela signifie que les espèces de lépidoptères sont en équilibre au niveau des différents types d'habitats étudiés.

Mots-clés: Parc National de Theniet El Had, Lépidoptères, Rhopalocères, Hétérocères, Diversité (H'), Equitabilité (E).

\section{How to cite this article:}

Kacha S, Adamou-Djerbaoui M, Marniche F, and De Prins W. The richness and diversity of lepidoptera species in different habitats of the national park theniet el had (Algeria). J. Fundam. Appl. Sci., 2017, 9(2), 746-769. 